\title{
ELECTROCHEMICAL STUDY OF THE DIFFUSION OF CUPRIC ION IN AQUEOUS AND AQUEOUS-GLYCEROL SOLUTIONS CONTAINING SULPHURIC ACID*
}

\author{
A. J. Arvia, J. C. BazÁn and J. S. W. Carrozza \\ Instituto Superior de Investigaciones, Facultad de Química y Farmacia, and División Ingenieria \\ Química, Facultad de Ciencias Físicomatematicas, Universidad Nacional de La Plata, La Plata, \\ Argentina
}

\begin{abstract}
The diffusion coefficient of cupric ion in sulphuric acid solutions containing glycerol from 0 to $10 \mathrm{M}$ has been determined with the aid of the rotating disk electrode. The viscosity of the solutions has also been measured. Experiments covered a temperature range from 18 to $40^{\circ} \mathrm{C}$. The experimental results are in agreement with the predictions of the Einstein-Stokes theory for diffusivity and viscosity. The ratio $D \eta / T$ is $(2.23 \pm 0.37) \times 10^{-10} \mathrm{~cm}^{2}$ poise/ $s^{\circ} \mathrm{K}$ and it is reasonably constant within the whole range of viscosity and temperature investigated.
\end{abstract}

Résumé-Le coefflcient de diffusion de l'ion cuivre(II) dans des solutions d'acide sulfurique contenant du glycérol d'une concentration de 0 à $10 \mathrm{M}$ a été déterminé au moyen d'une electrode à disque tournante. La viscosité des solutions a également été mesurée. Les expériences furent effectúés à une témperature entre 18 et $40^{\circ} \mathrm{C}$. Les résultats se rapprochent d'une façon satisfaisante des prédictions de la théorie d'Einstein-Stokes pour la diffusivité et la viscosité. Le rapport $D \eta / T$ est $(2,23 \pm 0,37) \times 10^{-10} \mathrm{~cm}^{2}$ poise $/ \mathrm{sec}^{\circ} \mathrm{K}$ et reste assez constant dans tout le champ de la viscosité et de la témperature étudiés.

Zusammenfassung-Mittels der rotierenden Scheibenelektrode wurde der Diffusionskoeffizient von Kupferion in schwefelsäure Lösungen bestimmt, welche zwischen 0 und $10 \mathrm{M} / \mathrm{L}$ Glycerin enthielten. Die Viskositāt dieser Lösungen wurde ebenfalls gemessen. Die Messungen erstreckten sich über den Temperaturbereich zwischen $18^{\circ}$ bis $40^{\circ} \mathrm{C}$. Die experimentell bestimmten Werte sind zufriedenstellend in Úbereinstimmung mit der Einstein-Stokes-Beziehung zwischen Diffusion und Viskosităt. Der Wert für das Verhältnis $D \eta / T$ ist $(2,23 \pm 0,37) \times 10^{-10} \mathrm{~cm}^{2}$ poise/sek $\mathrm{K}$, und bleibt im ganzen erfassten Temperatur- und Viskositätsbereich einigermassen konstant.

\section{INTRODUCTION}

AQUEOUS solutions containing copper sulphate and sulphuric acid are suitable for the study of the mechanism of ionic mass transfer in electrochemical systems. These solutions are particularly useful in evaluating the influence of viscosity because this property is easily changed over a large range by the addition of glycerol.

In spite of the frequent application of these solutions, the effective diffusion coefficient of cupric ion in copper sulphate and sulphuric acid solutions, a figure required to interpret ionic mass-transfer data, is known fairly well only for the case of solutions without glycerol. The only work reported in the literature is that of Gordon and $\mathrm{Cole}^{1}$ where the dependence of the diffusion coefficient of copper sulphate in aqueous solutions on the sulphuric-acid and copper-ion concentrations and temperature was evaluated.

More recently, Tobias and Fenech ${ }^{2}$ have published an equation for computing diffusion coefficients of the system containing copper sulphate, sulphuric acid, water and glycerol, where the product of the dynamic viscosity and the diffusion coefficient is given as a serial function of each component concentration. The equation is based upon data given by Eisenberg, Wilke and Tobias in a previous publication. ${ }^{3}$

* Manuscript received 21 December 1964; as amended, 3 August 1965. 
The experience gathered in this laboratory about these systems indicates that the above-mentioned equation yields high values of the diffusion coefficient; the larger the viscosity the greater is the discrepancy. For this reason we have thought it necessary to obtain sounder experimental information about the diffusion coefficients of cupric ion in those media, especially over a large range of viscosity.

\section{EXPERIMENTAL TECHNIQUE}

\section{Measuring method}

The effective diffusion coefficient of cupric ion has been determined by an electrochemical measurement based upon the evaluation of the limiting current density when the solution is electrolysed on a rotating disk electrode at different rates. The method is based on the application of the equation of the rotating disk electrode with electrolysis under diffusion control, according to the solution given by Levich.4

Experimental details of the method have been given in a previous publication. ${ }^{5}$ The only special characteristic of the present work worth mentioning is the type of electrode and the solutions employed. The cathode consisted of a copper disk of $3.0 \mathrm{~mm}$ in diameter embedded centrally in a Lucite rod $10 \mathrm{~mm}$ in diameter. The electrode was attached to the rotating shaft and the electrical contact was made by means of a bronze spring. A platinum disk electrode plated with copper was also used. Both electrodes presented a mirror surface when they were used in the experiments.

In the application of the rotating disk electrode for determining diffusion coefficients, if in the course of the reaction the electrode surface changes continuously, as occurs in the present case because of the copper deposition, roughness can build up on the electrode, invalidating the results. To eliminate this complication as far as possible the following experimental technique was applied: after an inspection of the full current/voltage curves obtained with one electrode in the whole range of the experimental conditions studied, a constant potential of about $400 \mathrm{mV}$ (depending on the system) measured against a symmetrical reference electrode was applied to a second cathode similar to the former one, and the current was read a few s afterwards. Under these circumstances, because of the rotation of the electrode, a steady state was reached very rapidly. With this procedure it was possible to use the same electrode at least for ten runs before any appreciable roughness could interfere in the measurements. Naturally, the effect depends on the type of solution employed: for solutions of the highest viscosity the electrode could be used longer without renewed polishing.

The reproducibility of limiting current densities was checked by changing the rotation rate of the working electrode upwards and downwards. The agreement of results was satisfactory within 3 per cent.

\section{Electrolytic solutions and working conditions}

Solutions were prepared with Merck p.a. copper sulphate and sulphuric acid. Purified glycerol obtained by distillation and ion exchange with an initial concentration of 98 per cent was used to obtain solutions of different viscosities.

Sulphuric acid was varied between 0.5 and $1.8 \mathrm{M}$, copper sulphate between 0.01 and $0.08 \mathrm{M}$ and glycerol between 1 and $10 \mathrm{M}$. The concentration of each of the species entering the solutions was evaluated by chemical analysis, the glycerol content being determined by titration with sodium metaperiodate. 
Experiments were performed in the range of temperature between 18 and $40^{\circ} \mathrm{C}$. Within the same range of temperature the viscosity and density of solutions were also determined.

\section{RESULTS}

A linear dependence of the limiting current density on the square root of the rotation velocity was obtained for all the experiments performed in the range of velocity between 20 and $120 \mathrm{rad} / \mathrm{s}$. Figure 1 presents experimental data

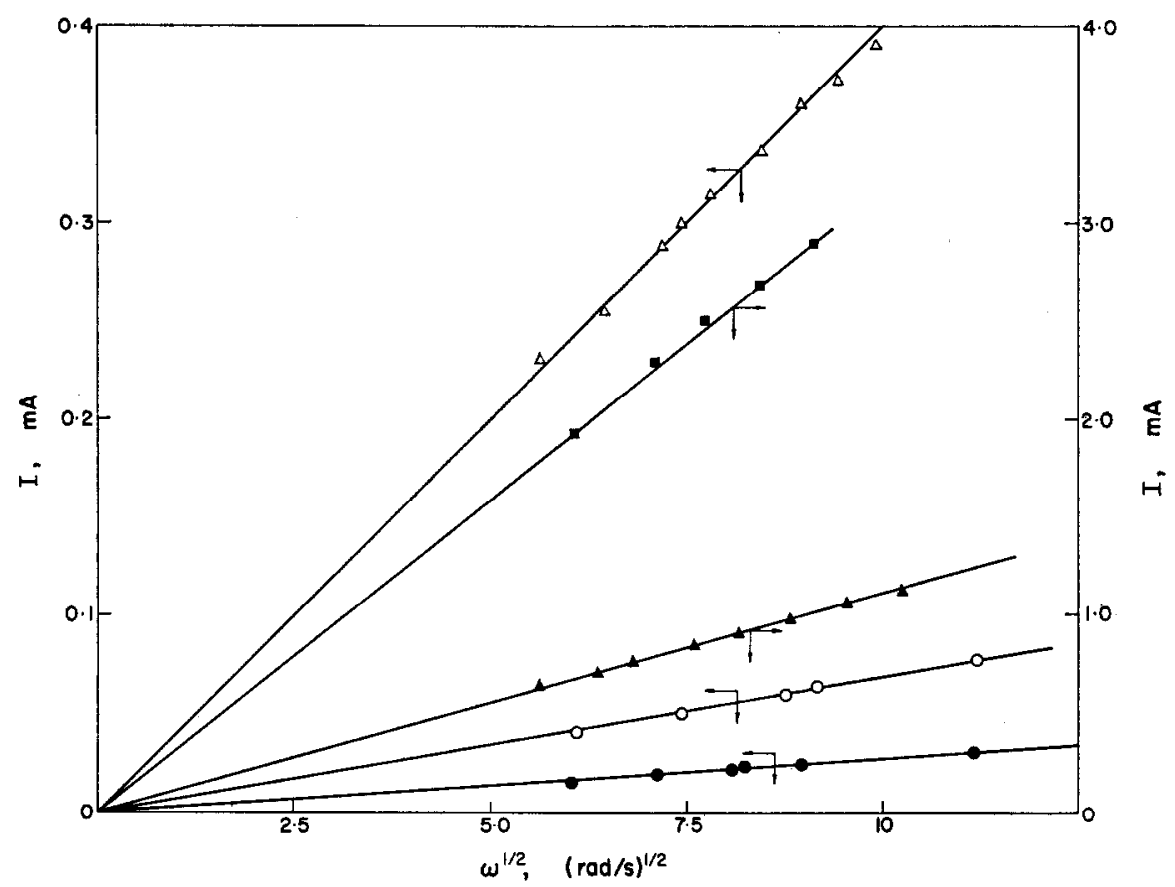

Fig. 1. Plot of $I_{\mathrm{L}}$ vs $\omega^{1 / 2}$ at $25^{\circ} \mathrm{C}$

- $C_{\mathrm{g}} 9.30 \mathrm{M} ; C_{\mathrm{a}} 1.431 \mathrm{M} ; C_{\mathrm{c}} 0.0100 \mathrm{M}$

O $C_{\mathrm{g}} 7.77 \mathrm{M} ; C_{\mathrm{a}} 1.463 \mathrm{M} ; C_{\mathrm{c}} 0.0100 \mathrm{M}$

$\triangle C_{\mathrm{g}} 1.94 \mathrm{M} ; C_{\mathrm{a}} 1.415 \mathrm{M} ; C_{\mathrm{c}} 0.0100 \mathrm{M}$

$C_{\mathrm{g}} 0.00 \mathrm{M} ; C_{\mathrm{a}} 1.693 \mathrm{M} ; C_{\mathrm{c}} 0.0250 \mathrm{M}$

- $C_{\mathrm{g}} 0.00 \mathrm{M} ; C_{\mathrm{a}} 1.752 \mathrm{M} ; C_{\mathrm{c}} 0.0770 \mathrm{M}$

obtained with solutions having different concentrations of their components. The slopes of the straight lines were used to evaluate the diffusion coefficients.

Results are assembled in Table 1 , where $C_{\mathrm{e}}, C_{\mathrm{a}}$ and $C_{\mathrm{g}}$ are respectively the molar concn. of copper sulphate, sulphuric acid and glycerol, $t$ is the temperature in degrees celsius, $\eta$ is the dynamic viscosity in poise, $d$ is the density in $\mathrm{g} / \mathrm{cm}^{3} ; S$ is the slope of straight lines resulting from the plots of the limiting current density $v s$ the square root of the rotation rate and $D$ is the diffusion coefficient of the reacting ion in $\mathrm{cm}^{2} / \mathrm{s}$. In the last column the ratio $D \eta / T$ is presented. With good approximation this figure is independent from temperature and viscosity of the solutions. Its average value is $(2 \cdot 23 \pm 0.37) \times 10^{-10} \mathrm{~cm}^{2}$ poise $/ \mathrm{s}^{\circ} \mathrm{K}$.

In Figs. 2 and 3 temperature dependence of the viscosity and the effective diffusion coefficient are plotted according to an Arrhenius relationship. Experimental activation 
TABle 1

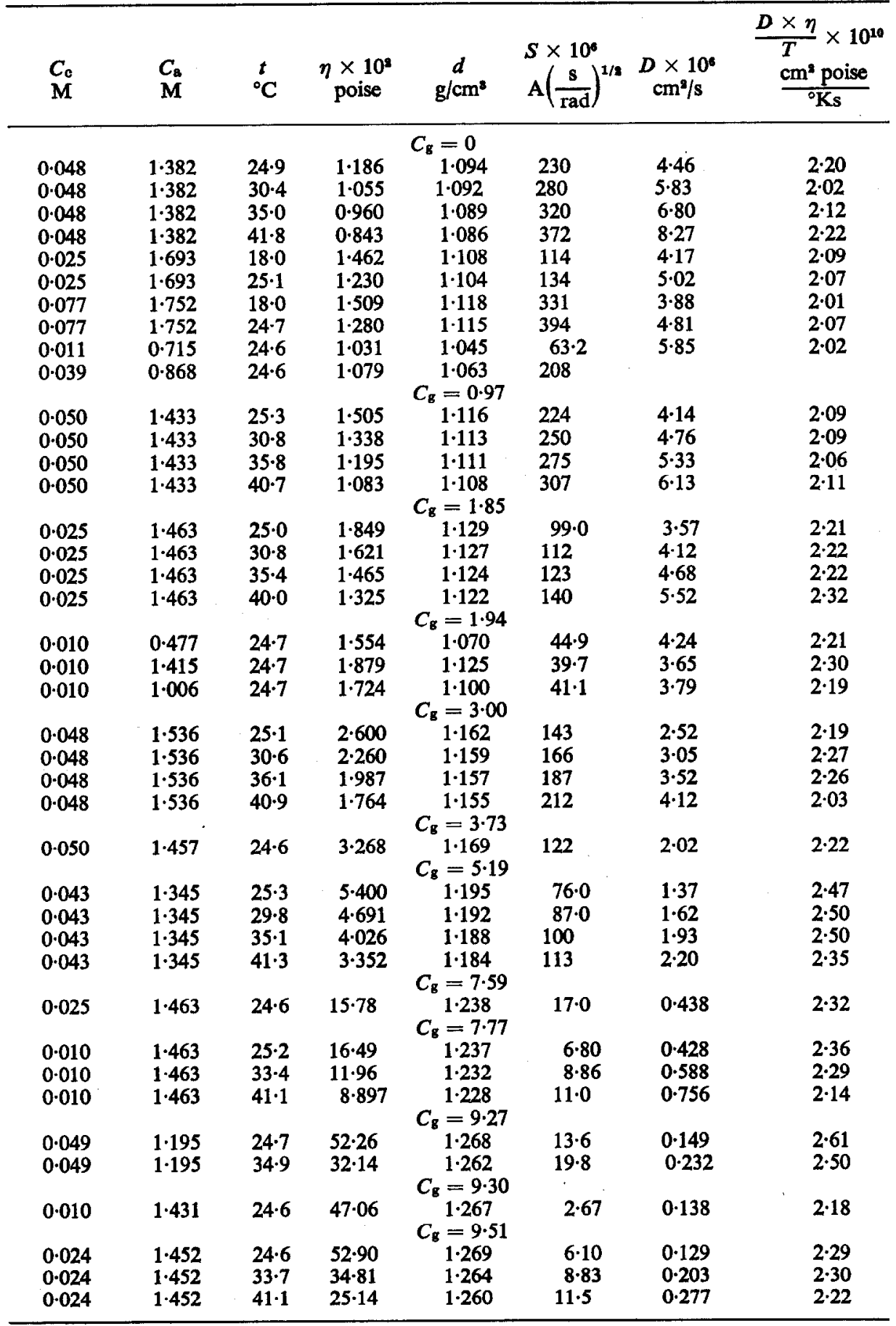




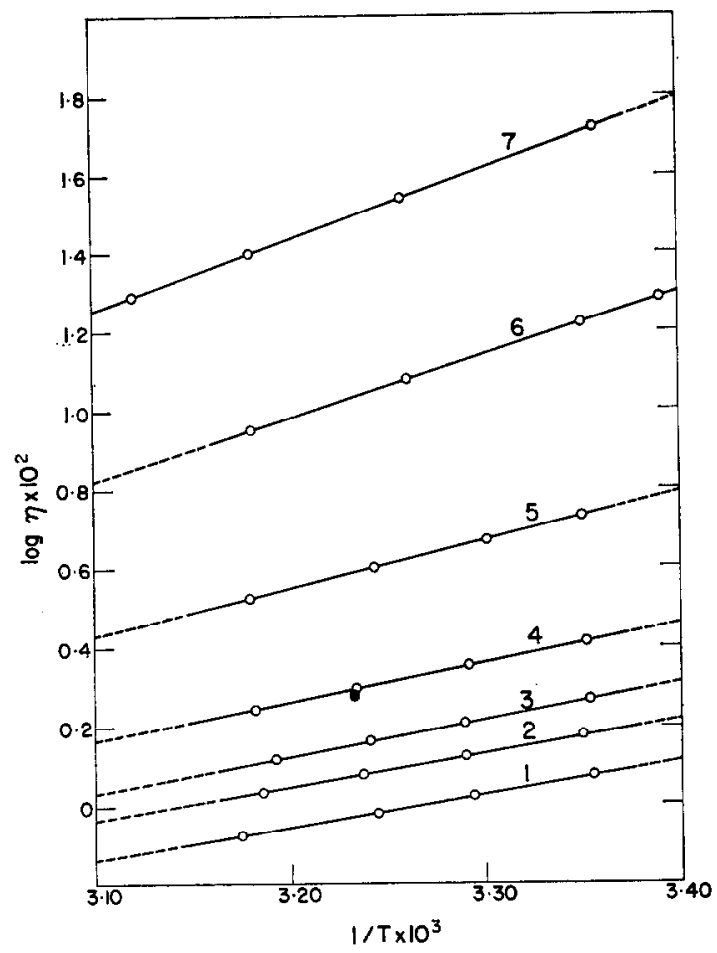

FIG. 2. Plot of $\log \eta v s 1 / T \times 10^{3}$

$\begin{array}{cccc} & C_{\mathrm{g}} & C_{\mathrm{a}} & C_{\mathrm{c}} \\ 1 & 0 & 1.382 & 0.0480 \\ 2 & 0.97 & 1.433 & 0.0500 \\ 3 & 1.85 & 1.463 & 0.0250 \\ 4 & 3.00 & 1.536 & 0.0480 \\ 5 & 5.19 & 1.345 & 0.0430 \\ 6 & 7.77 & 1.463 & 0.0100 \\ 7 & 9.51 & 1.452 & 0.0250\end{array}$

energies, $E_{\eta}$ and $E_{\mathrm{D}}$, are respectively obtained from those plots. For the case of viscosity a dependence of $E_{\eta}$ on the viscosity is clearly observed. The energy values in terms of fluidity cover the range of $3900 \pm 200 \mathrm{cal} / \mathrm{mol}$ for solutions without glycerol up to $8200 \pm 200 \mathrm{cal} / \mathrm{mol}$ for solutions nearly $10 \mathrm{M}$ in glycerol as shown in Fig. 4 . The same behaviour is observed for the diffusion coefficient although the temperature dependence is not so well established as in the former case, because the error involved in the determination is obviously larger.

Finally we must add that the effective diffusion coefficient for cupric ion was assumed to be the value for copper sulphate in the present case, since sulphate was the only anion present. So the data given by Gordon and Cole for the aqueous solutions of copper sulphate and sulphuric acid alone have been verified by performing the experiments under the same conditions used by these authors, and they satisfied within the experimental errors the constancy and the magnitude of the ratio $D \eta / T$.

\section{DISCUSSION}

The study of diffusion of cupric ion in aqueous sulphuric acid medium with the addition of glycerol shows very clearly that the product $D \eta / T$ is satisfactorily constant. 


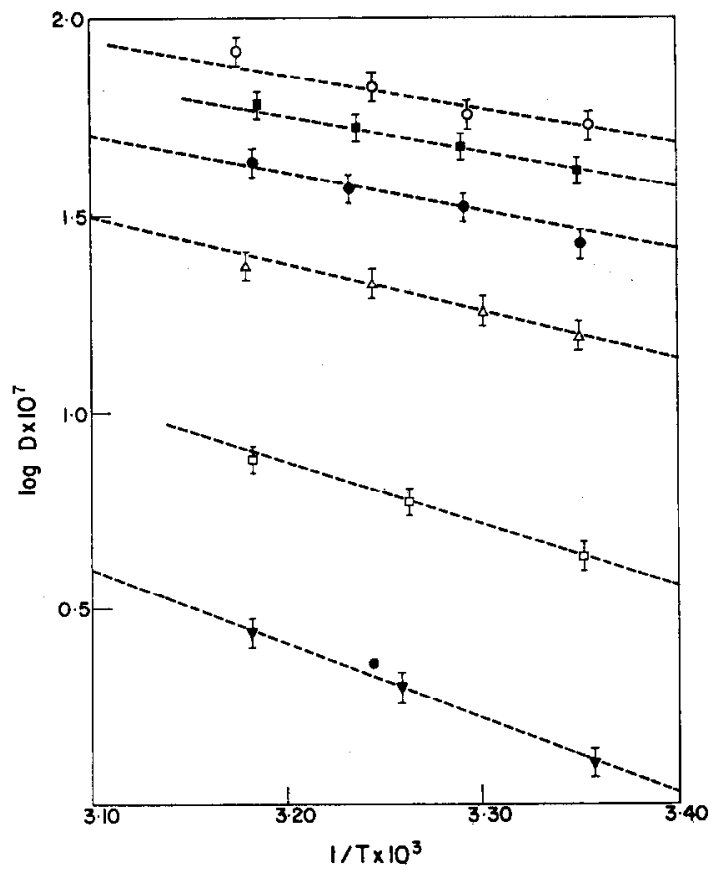

Fic. 3. Plot of $\log D \times 10^{7}$ vs $1 / T \times 10^{2}$

$\begin{array}{cccc} & C_{\mathbf{g}} & C_{\mathbf{z}} & C_{\mathbf{c}} \\ 0 & 0.00 & 1.382 & 0.0480 \\ & 0.97 & 1.433 & 0.0500 \\ \square & 3.00 & 1.536 & 0.0480 \\ \Delta & 5.19 & 1.345 & 0.0430 \\ \square & 7.77 & 1.463 & 0.0100 \\ \nabla & 9.51 & 1.452 & 0.0240\end{array}$

Dotted lines were drawn with the slopes of $\log \eta$ vs $1 / T$ for each solution.

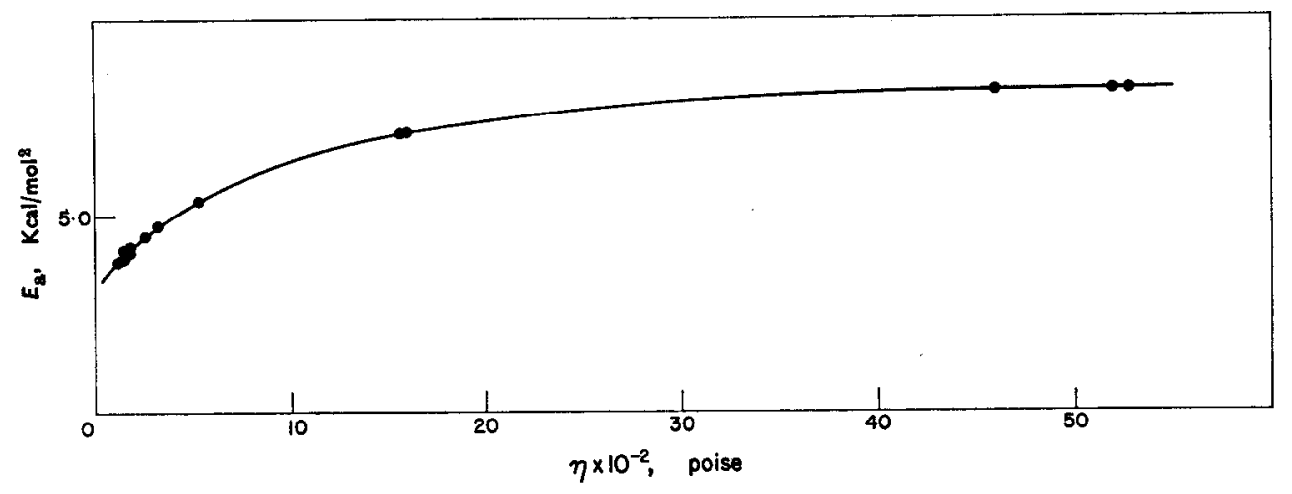

Fig. 4. Plot of $E_{\mathrm{s}}$ vs $\eta \times 10^{-9}$. 
In a previous report ${ }^{5}$ we noticed that this product was also constant for ferro- and ferricyanide ions in solutions containing different concentrations of sodium hydroxide, in spite of the complex nature of the solution, and any dependence of the diffusion coefficients on the composition was mainly assigned to change of viscosity of the system. The diffusion of ions under the present circumstances is analogous to the diffusion of neutral particles. This fact is confirmed by the results of the present work where a larger range of viscosity has been investigated.

It is remarkable that the experimental activation energy for the viscosity increases as the viscosity increases. This suggests that if the ratio $D \eta / T$ is a constant the same type of dependence should exist for the experimental activation energy for diffusion, as shown in Fig. 3. Furthermore, we can advance an interpretation of the foregoing results if diffusion and viscosity are considered from the standpoint of the theory of rate processes. ${ }^{6}$

If the main effect in the viscous and diffusional phenomena is due to the nature of the medium it is reasonable to assume that in the case of a constant ionic composition the increase of the activation energy is principally related to the nature of the solvent. However, the different solutions studied are certainly complex systems and the energy of activation must be related to various concurrent facts. According to the theory one of the energy terms is that required to create a hole in the liquid which amounts to an energy of the order of the enthalpy of vapourization. Another corresponds to the energy required for the molecule to move in the new hole. If ions are present in the solution ionic interactions and solvent-ion interactions must be taken into account. These interactions are larger when the ionic strength of the solutions increases as has been demonstrated in various cases. ${ }^{7}$

To eliminate these ionic interactions the diffusion coefficient at zero ionic strength has been estimated. This has been done by assuming that the ratio $\mathrm{D} \eta / T$ is only slightly dependent on the total ionic concentration because of the nature of the electrolyte and that at infinite dilution the viscosity of the system is the same as that of a glycerol-water solution. Thus, the activation energy for diffusion and viscosity can be more reasonably ascribed to the thermodynamic properties of the activated complex involved in the transport phenomena at infinite dilution. The equations established by transition-state theory for fluidity, $\phi$, and diffusion coefficient, $D$, are respectively

$$
\begin{gathered}
\phi=A^{\prime} \exp \left[-\frac{E_{\mathrm{a}}}{R T}\right]=\frac{V}{h N} \exp \left[\frac{\Delta S^{*}}{R}\right] \exp \left[-\frac{\Delta H^{*}}{R T}\right], \\
D=A^{\prime \prime} \exp \left[-\frac{E_{\mathrm{D}}}{R T}\right]=\lambda^{2} \frac{k T}{h} \exp \left[\frac{\Delta S^{*}}{R}\right] \exp \left[-\frac{\Delta H^{*}}{R T}\right], \\
E_{\mathrm{D}} \sim E_{\mathrm{a}} \sim \Delta H^{*} .
\end{gathered}
$$

In (1) and (2), $V$ is the apparent molar volume of the solution; $\Delta S^{*}$ and $\Delta H^{*}$ refer to the entropy and enthalpy of formation of the activated complex; $\lambda$ is a distance defined by the theory. $\Delta H^{*}$ was taken as equivalent to the experimental activation energy, $E_{\mathrm{a}}$. The rest of the symbols have the usual meanings. The pre-exponential factor was calculated and the entropy of formation of the activated complex was also obtained. The apparent molar volume of the solution was derived from the density tables for the glycerol-water solutions given in the literature. ${ }^{8}$ Entropy changes and 
free energies of formation of the activated complex, $\Delta G^{*}$, at infinite dilution are given in Table 2.

Both $\Delta S^{*}$ and $\Delta G^{*}$ are almost equally dependent on the composition of the solvent. It is unlikely that the increase of $\Delta S^{*}$ be ascribed to change of configuration of the diffusing species, because as it is further shown its apparent size appears on the average as independent from the composition of the solution. Then, the increase of entropy is an indication that the formation of the activated complex occurs simultaneously

TABle 2. Activation Parameters for the DIffusion PROCESS

\begin{tabular}{|c|c|c|c|}
\hline $\begin{array}{l}C_{\mathbf{g}} \\
\mathbf{M}\end{array}$ & $\begin{array}{c}E_{\mathrm{a}} \\
\mathrm{Kcal} / \mathrm{mol}\end{array}$ & $\underset{\mathrm{cal} / \mathrm{mol}^{\circ} \mathrm{K}}{\Delta S_{\mathrm{ags}}^{*}}$ & $\begin{array}{c}\Delta G_{2 \mathrm{as}}^{*} \\
\mathrm{Kcal} / \mathrm{mol}\end{array}$ \\
\hline 0.97 & 3.95 & 5.5 & $2 \cdot 31$ \\
\hline 1.85 & $4 \cdot 10$ & $5 \cdot 2$ & 2.55 \\
\hline 1.94 & $4 \cdot 20$ & $5 \cdot 7$ & $2 \cdot 50$ \\
\hline 3.00 & 4.50 & $6 \cdot 1$ & $2 \cdot 68$ \\
\hline 3.73 & $4 \cdot 80$ & 6.6 & $2 \cdot 83$ \\
\hline 5.19 & $5 \cdot 40$ & 7.6 & $3 \cdot 13$ \\
\hline $7 \cdot 59$ & $7 \cdot 10$ & $11 \cdot 3$ & $3 \cdot 73$ \\
\hline $7 \cdot 77$ & $7 \cdot 10$ & $11 \cdot 1$ & 3.79 \\
\hline $9 \cdot 27$ & $8 \cdot 20$ & $13 \cdot 2$ & $4 \cdot 27$ \\
\hline $9 \cdot 30$ & $8 \cdot 20$ & $13 \cdot 3$ & $4 \cdot 24$ \\
\hline 9.51 & $8 \cdot 20$ & 13.0 & $4 \cdot 33$ \\
\hline
\end{tabular}

with an increase of disorder in the system. This is reasonable because the molecular association of the solvent increases with the concn. of glycerol, involving a structural factor which affects the diffusion phenomena. Therefore, this effect must be related to the state of order of the particles in the initial and transition states.

The free energy of activation which determines the velocity of the process also increases with the concn. of glycerol at a given temperature. It might reasonably be supposed that it depends intimately on the specific process mechanism and its increase should be related to the larger association in the solvent as glycerol is added due to a corresponding increase of hydrogen bonding.

The constancy of the term $D \eta / T$ can be interpreted according to the following expressions given respectively by the Einstein-Stokes and the transition-state theory,

$$
\begin{gathered}
\frac{D \eta}{T}=\frac{k}{6 \pi r}, \\
\frac{D \eta}{T}=\frac{\lambda_{1}}{\lambda_{2} \lambda_{3}} k .
\end{gathered}
$$

In (4) $r$ is the average radius of the diffusing entity and in (5) $\lambda_{1}, \lambda_{2}$ and $\lambda_{3}$ are distances orthogonally taken, as postulated in the theory. From the average figure of $D \eta / T$, and taking $k=1.38 \times 10^{-16} \mathrm{erg} / \mathrm{deg}$. molecule, (4) yields $r=(3.28 \pm 0.60) \times$ $10^{-8} \mathrm{~cm}$, which is an acceptable figure for the average apparent radius of the diffusing ion. If (5) is applied, the average estimated $\lambda$ is $(0.62 \pm 0 \cdot 10) \times 10^{-6} \mathrm{~cm}$. It is difficult to explain this figure on a simple pictorial basis.

To conclude, although an interpretation of the energy terms has been given in terms of transition-state theory of transport phenomena, the latter is unsatisfactory as regards the Einstein-Stokes constant. 
Acknowledgements-This work was in part sponsored by the Consejo Nacional de Investigaciones Cientificas y Técnicas de la República Argentina. J. C. B. thanks the Consejo for the fellowship granted. Authors are indebted to the referees for their suggestions.

\section{REFERENCES}

1. A. R. Gordon and A. Cole, J. phys. Chem. 40, 733 (1936).

2. E. J. FENECH and C. W. TOBIAS, Electrochim. Acta 2, 311 (1960).

3. C. R. WILKE, M. Eisenberg and C. W. Tobias, J. electrochem. Soc. 100, 517 (1953).

4. B. Levich, Physicochemical Hydrodynamics. Prentice-Hall, Englewood Cliffs (1962).

5. J. C. BAZÁN and A. J. ARVIA, Electrochim. Acta 10, 1025 (1965).

6. S. Glasstone, K. J. LAIDLER and H. Eyring, The Theory of Rate Processes. McGraw-Hill, New York (1941); R. E. Powell, W. E. Roseveare and H. EYRING, Ind. Engng Chem. 33, 430 (1941).

7. G. Jones and M. Dole, J. Am. chem. Soc. 51, 2950 (1929).

8. International Critical Tables, Vol. III p. 121. McGraw-Hill, New York (1928). 